\title{
On-line acquisition of the output of a multi-channel analyzer (SMA 12/60) by means of an Elliott computer system
}

\author{
N. W. CARTER AND P. D. GRIFFITHS \\ From the University Department of Clinical Chemistry, The Royal Infirmary, Dundee
}

SYNOPSIS The introduction of a multi-channel analyzer (Technicon SMA 12/60) into a laboratory, in which the outputs from single-channel, continuous flow analyzers (Technicon AA1) are acquired on-line to an Elliott 903 computer system presented an additional problem. One possible solution is discussed.

In an earlier report (Griffiths and Carter, 1969) the on-line acquisition of the outputs from a number of Technicon AutoAnalyzer 1 channels by means of an Elliott 903 computer system was described. In 1969 an SMA 12/60 was installed to pursue a series of studies on behalf of the Eastern Regional Hospital Board (Scotland).

This machine produces 720 results on $40 \mathrm{ft}$ of chart paper every hour. Although the manufacturers intended the chart record (Fig. 1) to serve as a laboratory report, its size $\left(8 \frac{1}{4}\right.$ in. $\times 12 \frac{1}{4}$ in.) makes it unsuitable for incorporation into the medical records folder and, furthermore, the absence of a record of the results in the laboratory was another undesirable feature. It therefore seemed logical to utilize our Elliott computer system to tackle this data acquisition problem also.

Two possible methods of connecting the $12 / 60$ to the computer were considered. One was to connect the output of each of the 20 photocells via a separate buffer amplifier into the Elliott analogue multiplexer. The other was to fit a retransmitting slide wire to the chart recorder and feed one channel of an Elliott bench console. The first alternative was a major development involving the design and building of special amplifiers because the electrical characteristics of the 12/60 photocells are different from those used in AutoAnalyzers 1. Furthermore, the 12/60 electronics and programmer module would have become redundant. Therefore it was considered expedient in the first instance to adopt the simpler course.

Received for publication 13 January 1971.

\section{Methods}

HAR D W A R E

A small commercial reference voltage supply unit (provided by Technicon Instruments Co.) was affixed to the inside rear panel of the $12 / 60$ recorder cabinet. The output from this device is attenuated by a resistor network to a level of 8 millivolts and applied across a duplicate slide wire (Technicon Instruments Company) attached to the spindle of the $12 / 60$ recorder. This voltage, together with the signal from the wiper, feeds one position of the Elliott bench console to give a measure of the position of the pen on the chart recorder.

The frequency of the interrupts from the clock in the Elliott data acquisition unit was increased from once every two seconds to once every half second in order to obtain sufficient readings to validate and calculate the output from each 12/60 channel (12 per minute compared with one or one and a half minutes for each trace from Technicon AA1). The two second reading frequency for the Technicon AA1 was retained by means of software control.

\section{SOFTWARE}

To enable the $12 / 60$ to be handled at the same time as the single channels, the additional software had to be inserted into the existing structure. Apart from the routine for printing out blocks of results, entirely new software was required for 'peak' detection, calibration of results, and dealing with the operating procedures of the $12 / 60$ because (1) 12 'peak' crests are presented per minute from each specimen; (2) although the output from each estimation is switched to the recorder in a fixed sequence for each 
N. W. Carter and P. D. Griffiths

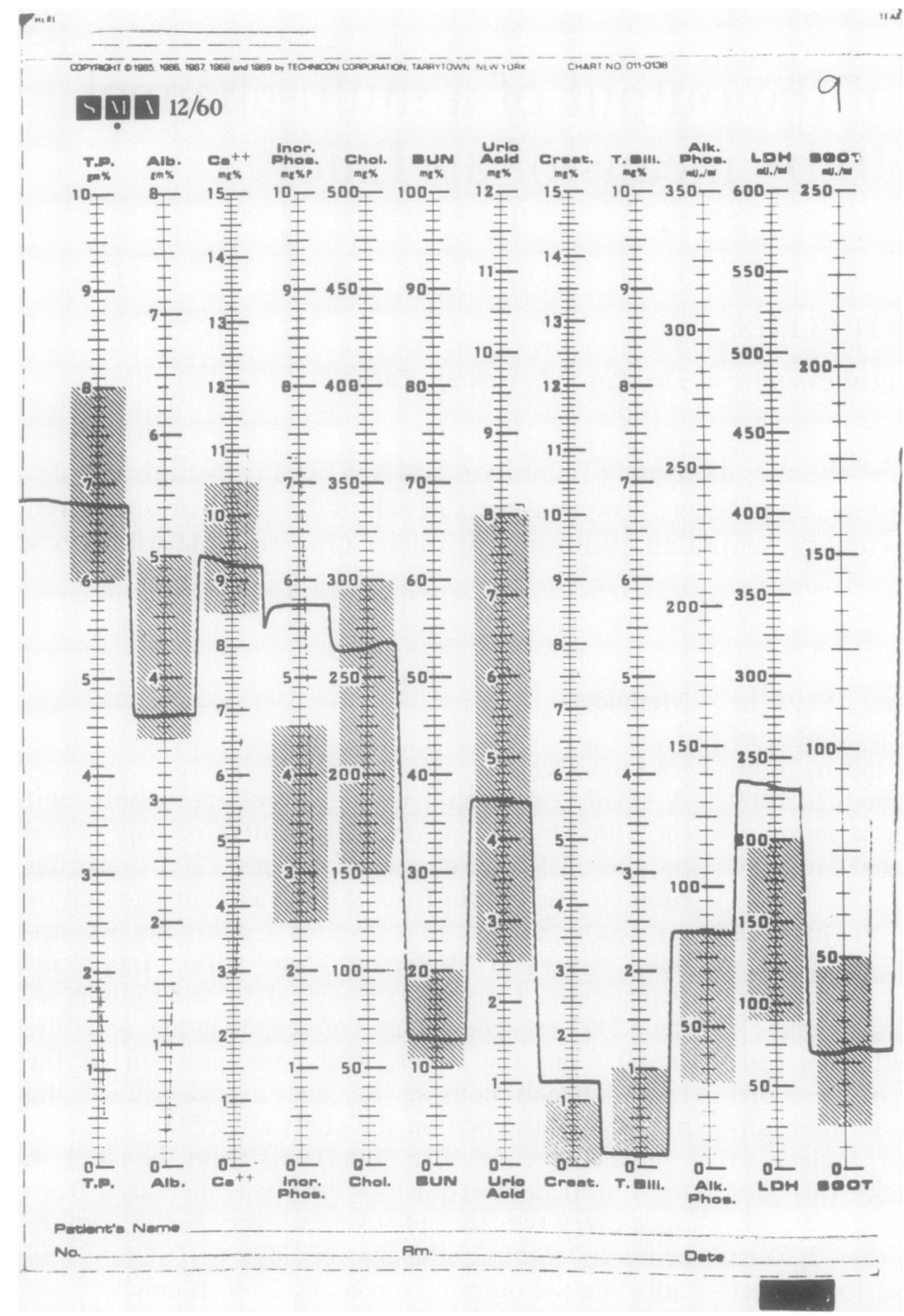

Fig. 1 Chart record of a 12-channel continuous flow analyzer (SMA 12/60). Actual size 12.25 in. $\times 8.25$ in.

sample, the trace from the first estimation lasts for nine seconds, the last trace six seconds, and the intervening 10 take four and a half seconds each; (3) this trace (called by Technicon 'steady state') represents only a small portion of the full peak shape, and of this an additional portion is usually lost during pen travel between consecutive traces; (4) it is not a true peak detection routine that is needed to select and validate the trace from each estimation. The pen may rise or fall at each end of the 'steady state' or even remain at the same level. Malfunctions and missing or aqueous samples often render consecutive traces indistinct from each other. Synchronization of the software is therefore difficult, assisted only by the $12 / 60$ time cycle and, when present, the start of pen travel following each trace; (5)calibration has to be performed using only one setting standardN of stated values for each estimation and a zerobaseline level. With the exception of the SGOT channel, linearity is claimed by the manufacturers.

Readings of the wiper and reference voltages from the duplicate slide wire are taken every half secondo and the ratio produced is stored in a circular buffer. $\Phi$ This ratio takes account of any drift in the reference? ${ }^{?}$ power supply. Once each minute the detection program analyses this buffer seeking a series of persistent values, ie, 'steady state', for each of the $12 \stackrel{\mathbb{}}{\overparen{Q}}$ channels. The acceptable values and markers for the $\mathbb{\mathrm { D }}^{\mathbb{Q}}$ 
rejected ones are then placed in a further circular buffer. As soon as the 60 values from a block of five specimens are available, they are converted into concentration values; then the results enter the output routine. Given a reading corresponding to the maximum and zero positions on the chart paper, it is a simple matter to use linear interpolation against the range of the linear concentration scale for each estimation. Although the SGOT scale on the chart paper is not completely linear, the same technique has been used against a linear scale which best approximates the SGOT scale. This has proved adequate for this channel, which can be regarded as little more than screening for a rough estimate of this enzyme's activity. Thus, being able to treat all channels alike, has much simplified the software. Values above and below the full scales are reported as $\mathrm{H}$ (high) and $\mathrm{L}$ (low) respectively, and unacceptable ones as $F$ (faulty).

\section{OPERATION}

The conversational mode of operation of the computer system has been extended by allowing the appropriate branch to offer fouralternative functions,
STR, BSE, RUN, and END. When setting up the $12 / 60$ the replies STR (set top range) and BSE (set base line) enable the program to take one reading from the pen set manually at the top and bottom of the chart paper scale respectively. These are the values used to demarcate the concentration scale for each estimation.

With the recorder running, the baseline of each channel is set to zero by means of the appropriate control on the $12 / 60$ programmer. The machine is then calibrated by sampling three cups of setting standard, adjusting to the stated values by means of the second set of controls on the programmer. Three cups of deionized water follow to bring the pen back to base line, and towards the end of these traces the reply RUN is typed in, thereby triggering the software which seeks out the beginning of the sample run. The software recognizes a threshold level for the first sample trace and once this has been passed, it starts to fill the primary buffer with readings. The first two samples are pooled sera, so that there is no possibility of patient samples being diluted by carryover from the cups containing water. When the run has been completed the reply END is typed in to

\begin{tabular}{|c|c|c|c|c|c|c|c|c|c|c|c|c|c|}
\hline & & TP & ALB & CA & PHD & ChO & BUN & URA & CRE & TBI & ALP & LDH & \\
\hline $\sin$ & 1 & 6.4 & 4.2 & 10.0 & 5.4 & 211 & 63 & 7.9 & 5.4 & 1.8 & 83 & 357 & \\
\hline $\sin$ & 2 & 6.6 & 4.3 & 9.9 & 5.6 & 265 & 66 & 8.0 & 5.3 & 1.8 & 85 & 369 & \\
\hline $\sin$ & 3 & 8.0 & 3.4 & 9.7 & 3.1 & 287 & 15 & 6.9 & 1.5 & 0.2 & 64 & $\mathbf{F}$ & \\
\hline $\sin$ & 4 & 7.9 & 3.5 & 9.4 & 3.4 & 253 & 20 & 7.2 & 1.1 & 0.1 & 177 & 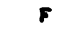 & \\
\hline $\sin$ & 5 & 6.6 & 2.7 & 8.7 & 2.3 & 317 & 9 & 4.9 & 0.8 & 0.1 & 84 & 159 & \\
\hline $\sin$ & 6 & 8.0 & 3.4 & 9.2 & 3.2 & 223 & 23 & 5.9 & 1.1 & 0.2 & 120 & $\boldsymbol{F}$ & \\
\hline $\sin$ & 7 & 6.7 & 3.1 & 8.5 & 3.0 & 315 & 25 & H & $\boldsymbol{F}$ & 0.2 & 64 & 352 & \\
\hline $\sin$ & 8 & 6.6 & 2.9 & 8.5 & 6.3 & 236 & 50 & 6.9 & 2.8 & 0.4 & 70 & $\boldsymbol{F}$ & \\
\hline $\sin A$ & 9 & 6.8 & 3.7 & 9.3 & 5.8 & 268 & 13 & 4.5 & 1.3 & 0.2 & 85 & 233 & \\
\hline MA & 10 & 7.3 & 4.3 & 9.5 & 3.6 & 239 & 19 & 4.4 & 1.1 & 0.2 & 76 & 169 & \\
\hline mA & 11 & 7.6 & 4.7 & 9.8 & 4.4 & 248 & 13 & 5.0 & 1.0 & 0.2 & 47 & 217 & \\
\hline 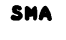 & 12 & 6.8 & 4.5 & $\mathbf{F}$ & 5.7 & 268 & 65 & 8.0 & 5.3 & 1.8 & 86 & F & \\
\hline sma & 13 & 7.5 & 4.3 & 9.3 & 3.6 & 263 & 17 & 5 & 5 & 0.2 & 7 & 173 & \\
\hline MA & 14 & 7.1 & 4.5 & 9.2 & 2.9 & 283 & 21 & 7 & 1.0 & 0.3 & 69 & F & \\
\hline A & 15 & 7.1 & 3.9 & 9.6 & 3.5 & 307 & 23 & 7.8 & 1.1 & 0.4 & 83 & 158 & \\
\hline ma & 16 & 6.4 & 4.3 & 200 & 3.9 & 262 & 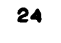 & 200 & 1.2 & 0.2 & 65 & 227 & \\
\hline M & 17 & 6.8 & 4.7 & 9.3 & H & 325 & 23 & 2.3 & 1.4 & 0.5 & 39 & 227 & \\
\hline$m$ & 18 & 7.7 & 3.4 & 9.1 & 2.9 & 250 & 33 & 7.9 & 1.4 & $0: 4$ & 56 & 258 & \\
\hline $\sin$ & 19 & 7.1 & 4 & 6 & 2.8 & 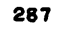 & 29 & 6.5 & 7 & 0.3 & 59 & $F$ & \\
\hline $\sin$ & 20 & 8.7 & 5.4 & 10.4 & H & 322 & 26 & 10.8 & 2.3 & 0.7 & 43 & 205 & \\
\hline
\end{tabular}

Fig. 2 Computer printout from a 12-channel continuous flow analyzer (SMA 12/60) using an Elliott 903 system. $H=$ high, $L=$ low, $F=$ faulty peak. 
allow the $12 / 60$ software to be by-passed. About 500 extra locations of the computer store were needed to accommodate the $12 / 60$ program.

\section{Discussion}

Incorporating the SMA 12/60 into an existing system of on-line acquisition from single channel AutoAnalyzers proved more difficult than at first was anticipated. Some form of data logging system is essential if the chart is not regarded as an acceptable means of reporting results to the clinician. The printout (Fig. 2) provided by the system described here can be affixed (or transcribed) to a normal report form, and presents one possible solution for laboratories already equipped with computer facilities.

It will be noted that no calculations to allow for such factors as drift and carry-over have been incorporated into the program. This was deliberate policy as part of the studies mentioned earlier involved assessment of these factors. These studies will be reported on another occasion. The manufacturers recommend that the calibrating standard should be run in every tenth cup and the technician has the tedious task of resetting each channel at this stage. A correction, using linear interpolation, can easily be incorporated into the program, but this step has been omitted as a preliminary assessment suggests that recalibration and/or computer correction is not a valid procedure.

One must accept that the use of the duplicate slide $\overrightarrow{\vec{F}}$ wire is little more than an expedient. It has enabled us to obtain more easily the output from a multichannel analyzer in a form suitable for both analysis $\frac{\sigma}{\omega}$ and onward transmission. However, it faithfully $\vec{\nabla}$ reproduces only the graphic wave form christened by the manufacturers 'steady state'. In other words, $\infty$ we only see the results from a chemical analytical $\vec{\circ}$ process which has been subsequently modified by linearizing and other logic circuits. It will be necessary $\omega_{\mathscr{\omega}}$ to examine the colorimeter outputs directly to achieve $\frac{2}{O}$ a more critical evaluation of the machine. This will be an expensive experiment, but it is one which we believe should be carried out. All too little is known about continuous flow analysis at present and the $\stackrel{\circ}{\circ}$ advent of the new generation of continuous flow $\overrightarrow{0}$ analyzers intended for faster running presents a further challenge. The easier acquisition, and sub- $\overrightarrow{0}$ sequent analysis, of laboratory data, made possible by the introduction of computers into the laboratory, 응 can only lighten the daunting task of providing an $\stackrel{\Phi}{\simeq}$ accurate and reliable laboratory service.

Reference

Griffiths, P. D., and Carter, N. W. (1969). On-line acquisition of the output of AutoAnalyzers. J. clin. Path., 22, 609-616. 